\title{
Medición de costo de transacción tributaria en medianas empresas de la ciudad de Riobamba, período 2019
}

Measurement of tax transaction cost in medium-sized companies in the city of Riobamba, period 2019

1 Diego Alexander Calva Merino https://orcid.org/0000-0002-7824-0034 Universidad Nacional de Chimborazo, Facultad de Ciencias Políticas y Administrativas dacalva.fpa@unach.edu.ec

2 Gema Viviana Paula Alarcón Universidad Nacional de Chimborazo, Facultad de Ciencias Políticas y Administrativas gemapaula@unach.edu.ec

Artículo de Investigación Científica y Tecnológica

Enviado: 06/11/2021

Revisado: $21 / 11 / 2021$

Aceptado: 08/12/2021

Publicado:05/01/2022

DOI: https://doi.org/10.33262/visionariodigital.v6i1.1957

\section{Cítese:}

Calva Merino, D. A., \& Paula Alarcón, G. V. (2022). Medición de costo de transacción tributaria en medianas empresas de la ciudad de Riobamba, período 2019. Visionario Digital, 6(1), 6-24. https://doi.org/10.33262/visionariodigital.v6i1.1957

\footnotetext{
VISIONARIO DIGITAL, es una Revista Científica, Trimestral, que se publicará en soporte electrónico tiene como misión contribuir a la formación de profesionales competentes con visión humanística y crítica que sean capaces de exponer sus resultados investigativos y científicos en la misma medida que se promueva mediante su intervención cambios positivos en la sociedad. https://visionariodigital.org

La revista es editada por la Editorial Ciencia Digital (Editorial de prestigio registrada en la Cámara Ecuatoriana de Libro con No de Afiliación 663) www.celibro.org.ec
}

Esta revista está protegida bajo una licencia Creative Commons AttributionNonCommercialNoDerivatives 4.0 International. Copia de la licencia: http://creativecommons.org/licenses/by-nc-nd/4.0/. 


Palabras
claves: costo,
transacción
tributaria,
medianas
empresas,
costos de
administrativos,
costos cumplimiento,
contabilidad.

Keywords:

cost, tax transaction, medium-sized companies, administrative costs, compliance costs, accounted.
Resumen

Introducción. El Ecuador ha venido mejorando cada vez más en el funcionamiento de su sistema tributario a través de la creación de leyes y reglamentos para conseguir una correcta contribución por parte de los sujetos pasivos. Por lo tanto, se ha implementado diferentes mecanismos que permitan reducir costos tributarios en la declaración y recaudación de los diferentes impuestos que se generan en nuestro país. Sin embargo, pese a los constantes esfuerzos por parte de las administraciones tributarias para lograr dicha reducción, aún existen distintos costos implícitos que los sujetos pasivos deben efectuar para cumplir con sus obligaciones tributarias, lo cual genera que los contribuyentes incurran en la evasión, elusión e informalidad tributaria. Objetivo. Determinar la medición de costo de transacción tributaria de las medianas empresas de la ciudad de Riobamba en el período 2019. Metodología. El método aplicado fue deductivo, con un enfoque cualitativo y cuantitativo, con un diseño no experimental y de tipo documental. La población fue de 1.461 contribuyentes y aplicando un cuestionario a una muestra de 230 contribuyentes. Resultados. Entre los resultados obtenidos en la investigación mediante la aplicación del método de regresión lineal muestran que la medición de transacción tributaria incide significativamente en las medianas empresas de la ciudad de Riobamba. Conclusión. Se concluyó que la utilización de la metodología de medición de costo de transacción tributaria es una buena herramienta para la medición de costos de los contribuyentes y el Servicio de Rentas Internas.

\section{Abstract}

Introduction. Ecuador has been increasingly improving the functioning of its tax system through the creation of laws and regulations to achieve a correct contribution by taxpayers. Therefore, different Introduction. Ecuador has been increasingly improving the functioning of its Tax System through the creation of laws and regulations to achieve a correct contribution by taxpayers. Therefore, different mechanisms have been implemented to reduce tax costs in the declaration and collection of the different taxes generated in our country. However, despite the constant efforts made by the Tax Administrations to achieve such reduction, there are still different implicit costs that taxpayers must incur to comply with their tax obligations, which causes taxpayers to incur in tax 
evasion, avoidance and informality. Objective. To determine the tax transaction cost measurement of medium-sized companies in the city of Riobamba for the 2019 period. Methodology. The applied method was deductive, with a qualitative and quantitative approach, with a non-experimental and documentary type design. The population was 1,461 taxpayers and applying a questionnaire to a sample of 230 taxpayers. Results. The results obtained in the research through the application of the linear regression method show that the tax transaction measurement has a significant impact on medium-sized companies in the city of Riobamba. Conclusion. It was concluded that the use of the tax transaction cost measurement methodology is a good tool for measuring the costs of taxpayers and the Internal Revenue Service.

\section{Introducción}

La obligación tributaria es el vínculo jurídico personal que deben cumplir tanto las personas físicas como jurídicas frente a la administración tributaria, que en nuestro país se trata del Servicio de Rentas Internas, entidad que determina las directrices para que los contribuyentes cumplan con todos sus deberes formales (Código Tributario, 2019). Por lo tanto, todas las medianas empresas de la ciudad de Riobamba están en la obligación de declarar todos los impuestos concernientes de las actividades por ellas ejecutadas, y de esta manera poder obtener mejores resultados finales al culminar su ejercicio económico. Por ello, el propósito del trabajo de investigación es la medición de costo que conlleva el cumplimiento de la transacción tributaria en estas empresas, verificando que hayan declarado correctamente todos los impuestos que de acuerdo con lo estipulado en las disposiciones legales vigentes están sujetas a cumplir.

Con el paso de los años la administración tributaria se ha venido esforzando cada vez más por aumentar la recaudación de los impuestos, reduciendo las brechas de inscripción, presentación, veracidad y recaudación de los impuestos tributarios, por medio de la creación de reformas tributarias y la gestión eficiente de los funcionarios de la administración tributaria; por lo tanto, debido a los constantes cambios que se han producido en la normativa tributaria y a la compleja aplicación del sistema tributario que se produce para los varios contribuyentes, ha generado que dichos sujetos pasivos incurran en diferentes gastos adicionales en el cumplimiento de su obligaciones tributarias. Es así, que en la ciudad de Riobamba no se ha realizado un estudio que permita obtener información real en relación con la transacción tributaria que incurren los contribuyentes, en este caso las medianas empresas al cumplir sus obligaciones 
tributarias. Por lo tanto, es indispensable realizar una investigación que posibilite comprender y cuantificar los costos monetarios, y con ello el costo de oportunidad que corresponden a los contribuyentes de la ciudad de Riobamba.

Con relación a los resultados que se obtendrán con la investigación esta contribuir a la Administración Tributaria buscar y optar por nuevos mecanismos que permitan reducir ciertos costos de transacción tributaria a las medianas empresas, con el propósito que las mismas pueden centrarse en la generación de riqueza, contribuir al desarrollo económico del país, y así evitar y reducir la elusión y evasión tributaria.

Una vez revisado las distintas fuentes bibliográficas de los trabajos de investigación relacionadas se puede identificar que El Centro Inter americano de Administraciones Tributarias, en su trabajo de investigación titulado "Medición de los Costos de Transacción Tributarios en Pequeñas y Medianas Empresas", concluye que la implementación de la metodología de medición de los costos de transacción tributarios es el punto de partida para que la Dirección General Tributaria tenga una herramienta de evaluación de los costos que incurre el contribuyente y la administración tributaria (Centro Inter-americano de Naciones Unidas, 2015, p. 263).

Por otra parte, Vargas (2017) en su trabajo de investigación de maestría titulado "El costo de la transacción tributaria y el cumplimiento de las obligaciones fiscales en las pequeñas y medianas empresas, sector comercio de la provincia de Tungurahua" (p.127). Concluye que el Ecuador ha diseñado a través de su página web herramientas que permitan disminuir el costo de la transacción tributaria, evitando la atención presencial y generando información en línea, a través de la web se pueden realizar consultas, generar certificados, solicitar devoluciones, pero principalmente se pueden remitir las declaraciones y anexos, incluso con una declaración propuesta para el caso de Impuesto a la Renta de personas naturales no obligadas.

Según Mera et al. (2017), en su artículo titulado "El costo de la transacción tributaria en las empresas comerciales de la Provincia de Tungurahua" (pp. 21-22). Concluyen que existe una diversidad de obligaciones entre declaraciones de impuestos y anexos, sin embargo no todas recaen sobre el mismo sujeto pasivo, por lo general la mayor parte de contribuyentes está afecto al Impuesto al Valor Agregado e Impuesto a la Renta en cuanto al tributo, y en cuanto a la entrega de información al anexo transaccional ATS y anexo en relación de dependencia, las compañías adicionalmente, por una sola vez al año, presentarán información relativa a sus accionistas.

Así al hablar de medición se establece que, "es el proceso de determinación de los importes monetarios por los que se reconocen y llevan contablemente los elementos de los estados financieros" (Laporta, 2016, p. 67). 
Según lo manifestado por Arredondo (2015) costo "es el desembolso económico que implica la oferta de un servicio o la elaboración de un producto" (p. 26).

Por lo tanto, la medición de costo de transacción tributaria es el proceso mediante el cual se aplica una fórmula que permite determinar los costos de cumplimiento y costos administrativos de un periodo determinado.

Según Vargas (2017) existe una serie de fases que permiten la medición del costo de transacción tributaria, los mismos se detallan a continuación:

Fase 0. Inicial. - en esta fase se analiza toda la información necesaria de la normativa tributaria que rige a las empresas analizadas en cuanto a sus obligaciones tributarias.

Fase 1. Preparatoria. - en esta fase se identifican los instrumentos y recursos para la ejecución del estudio.

Fase 2. Análisis. - en esta fase se identifica toda la información necesaria para la ejecución del estudio.

Fase 3. Medición. - aquí se efectúa la medición de los costos de cumplimiento y los costos de administración.

Fase 4. Informe. - es la etapa final donde ya se conocen los resultados de la fase anterior y se proponen estrategias que ayuden al cumplimiento de las obligaciones tributarias de estas empresas.

Según Castillo \& Vásquez (2015), los costos de transacción tributaria: “es la suma de los costos de administración tributarios y los costos incurridos en el cumplimiento tributario" (p. 11).

Por ello al mencionar sobre los Costos de Administración Tributarios se establece que: "son los costos asumidos por la autoridad tributaria a fin de cumplir cabalmente con sus responsabilidades de administrar el sistema tributario" (Laporta, 2016, p. 32).

En cambio, Montás (2018) manifiesta que: "los costos de cumplimiento tributario son aquellos costos en que los contribuyentes deben incurrir para cumplir con la legislación tributaria vigente" (p. 12).

Por consiguiente, se habla acera de las medianas empresas consideradas como: "toda persona natural o jurídica que, como una unidad productiva, ejerce una actividad de producción, comercio y/o servicios, y que cumple con el número de trabajadores y valor bruto de las ventas anuales" (Código Orgánico de la Producción, 2020, p. 26). Y de acuerdo con el análisis de los artículos 53 y 56 del Código Orgánico de la Producción, 


\section{VPDigital}

Comercio e Inversiones se clasifica a las PYMES en base a dos indicadores, como se muestra en el siguiente cuadro:

Tabla 1

Clasificación de las PYMES

\begin{tabular}{lll}
\hline Detalle & $\mathrm{N}^{\circ}$ de trabajadores & Ventas anuales \\
\hline Microempresa & 1 a 9 & Hasta $100,000.00$ \\
Pequeña empresa & 10 a 49 & $100,001.00$ a $1,000,000.00$ \\
Mediana empresa & 50 a 199 & $1,000,000.00$ a $5,000,000.00$ \\
\hline
\end{tabular}

Fuente: Código Orgánico de la Producción (2020)

\section{Metodología}

En la investigación se empleó el método deductivo según Gutiérrez (2016) afirma que el método deductivo "es el método que permite pasar de afirmaciones de carácter general a hechos particulares" (p. 24). Debido a que se empleó la encuesta para obtener información de las medianas empresas, además se aplicó una fórmula para la determinación del costo de transacción tributaria.

Además, cuenta con un diseño no experimental, tipo documental y con un nivel descriptivo, por lo que, describen situaciones y hechos acerca del tema tratado, en este caso el costo que incurre al momento de efectuar la transacción tributaria.

La población considerada para el estudio de la investigación corresponde a los 1.461 contribuyentes obtenidos de la base de datos del Servicio de Rentas Internas, los mismos que corresponden a las personas jurídicas obligadas a llevar contabilidad que se encuentran en estado activo de su RUC.

Se aplicó el muestreo probabilístico a través de la fórmula de cálculo para poblaciones finitas, determinando el tamaño de la muestra aleatoria simple.

$$
\begin{gathered}
n=\frac{z^{2} p(1-p) N}{e^{2}(N)+Z^{2} p(1-p)} \\
n=\frac{(1,65)^{2}(0,5)(1-0,5)(1461)}{(0,05)^{2}(1461)+(1,65)^{2}(0,5)(1-0,5)} \\
n=230
\end{gathered}
$$

Cálculo del tamaño de la muestra (López, 2015) 
- $\mathrm{n}$ : Tamaño de la muestra.

- $\quad \mathrm{N}: 1461$

- $\mathrm{Z}:=1,65$ (Nivel de confianza; para el 90\%)

- $\mathrm{p}:=0,5$ (Posibilidad de ocurrencia de un evento)

- $\mathrm{q}:=0,5($ Posibilidad de no ocurrencia de un evento)

- $\mathrm{E}:=0,05$ (Error de la estimación, se considera el 5\%)

La recolección de datos se efectuó a través de la técnica de la encuesta (Rodríguez y Pérez, 2017), y como instrumento se empleó el cuestionario que está conformado por 26 preguntas cerradas aplicada a las medianas empresas de la ciudad de Riobamba. Conforme al índice alfa de Cron Bach, el cuestionario tiene un grado de confiabilidad del $78,9 \%$.

\section{Resultados}

\section{Caracterización de las medianas empresas}

En cuanto al régimen al cual pertenecen las empresas encuestadas, el 100\% corresponde al régimen general, debido a que son entidades que están legalmente constituidas y sus actividades contables y tributarias son extensas.

Tabla 2

Régimen Tributario

\begin{tabular}{lccc}
\hline & & Frecuencia & Porcentaje \\
\hline Válidos & Régimen General (RG) & 230 & $100 \%$ \\
\hline & Total & 230 & $100 \%$
\end{tabular}

Nota: elaborado en base a la encuesta aplicada a las medianas empresas de Riobamba, año 2021

Como resultado de la encuesta aplicada a las medianas empresas tenemos que el $63.04 \%$ se ha capacitado por 7 días sobre obligaciones tributarias, el $36.96 \%$ lo hizo únicamente por 3 días y ninguna lo realizo por más de una semana, por lo cual, estos contribuyentes tienen claro cuáles son sus obligaciones con la Administración Tributaria, por ende, conocen cuales serían las sanciones del incumplimiento de estas, como se muestra en la tabla 3 . 


\section{Tabla 3}

Tiempo de Capacitación

\begin{tabular}{cccc}
\hline & & Frecuencia & Porcentaje \\
\hline Válidos & 3 días & 85 & 37,0 \\
& 7 días & 145 & 63,0 \\
\hline & Total & 230 & 100,0 \\
\hline
\end{tabular}

Nota: elaborado en base a la encuesta aplicada a las medianas empresas de Riobamba, año 2021

\section{Costos Internos}

En la investigación se plantearon varias interrogantes relacionadas a los costos internos que incurren las medianas empresas durante el año 2019, los cuales son factores indispensables al momento de la determinación de los costos de cumplimiento, por lo tanto, se detallan a continuación en la tabla 4:

\section{Tabla 4}

\section{Costos Internos}

\begin{tabular}{|c|c|c|c|}
\hline Pregunta & Media & $\begin{array}{l}\text { Desviación } \\
\text { Estándar }\end{array}$ & $\begin{array}{l}\mathrm{N} \text { de } \\
\text { análisis }\end{array}$ \\
\hline $\begin{array}{l}\text { P1. ¿Hace cuánto tiempo formalizó su empresa para } \\
\text { realizar sus actividades económicas? }\end{array}$ & 2,61 & 4,88 & 230 \\
\hline P2. ¿Cuánto fue el gasto notarial al constituir su empresa? & 1,67 & 4,73 & 230 \\
\hline $\begin{array}{l}\text { P3. ¿Cuál fue el tiempo que utilizo para la inscripción y } \\
\text { obtención del Registro Único de Contribuyentes? }\end{array}$ & 1,01 & 1,14 & 230 \\
\hline $\begin{array}{l}\text { P4. ¿Resulta oneroso el gasto en el proceso de ingreso en } \\
\text { formularios e inscripción del negocio en la } \\
\text { administración tributaria? }\end{array}$ & 1,00 & 0,000 & 230 \\
\hline $\begin{array}{l}\text { P5. ¿Cuál fue el tiempo utilizado al año para el registro } \\
\text { de sus operaciones contables? }\end{array}$ & 1,46 & 5,80 & 230 \\
\hline P6. ¿Qué tipo de comprobante de pago emite? & 2,09 & 2,82 & 230 \\
\hline $\begin{array}{l}\text { P7. ¿Cuánto gastó al año en la impresión de C/P (facturas, } \\
\text { notas de venta, guía de remisión, notas de crédito y débito } \\
\text { y liquidaciones) por millar? }\end{array}$ & 1,30 & 5,62 & 230 \\
\hline $\begin{array}{l}\text { P8. ¿Cuál fue el tiempo utilizado al año para el cálculo y } \\
\text { declaración de sus impuestos? }\end{array}$ & 1,16 & 4,30 & 230 \\
\hline
\end{tabular}




\section{Tabla 4}

Costos Internos (continuación)

\begin{tabular}{|c|c|c|c|}
\hline Pregunta & Media & $\begin{array}{l}\text { Desviación } \\
\text { Estándar }\end{array}$ & $\begin{array}{c}\mathrm{N} \mathrm{de} \\
\text { análisis }\end{array}$ \\
\hline $\begin{array}{l}\text { P9. ¿Cuál fue el tiempo utilizado al año para el pago de } \\
\text { sus impuestos? }\end{array}$ & 1,11 & 3,12 & 230 \\
\hline $\begin{array}{l}\text { P10. ¿Ha tenido alguna acción de control por parte del } \\
\text { Servicio de Rentas Internas durante los últimos } 12 \\
\text { meses? }\end{array}$ & 1,00 & 0,000 & 230 \\
\hline $\begin{array}{l}\text { P11. ¿En el caso de su respuesta ser afirmativa, cual fue } \\
\text { el tiempo que llevo la acción de control por parte del } \\
\text { SRI al año? }\end{array}$ & 1,11 & 3,12 & 230 \\
\hline $\begin{array}{l}\text { P12. ¿Ha efectuado algún tipo de reclamo y solicitud de } \\
\text { devolución de impuestos durante los últimos } 12 \text { meses? }\end{array}$ & 1,09 & 2,82 & 230 \\
\hline $\begin{array}{l}\text { P13. ¿En el caso de su respuesta ser afirmativa, cual fue } \\
\text { el tiempo que llevo a cabo para efectuar el reclamo o } \\
\text { devolución al año? }\end{array}$ & 1,32 & 8,82 & 230 \\
\hline $\begin{array}{l}\text { P14. ¿Cuáles son los gastos administrativos anuales que } \\
\text { se producen en su empresa para el cumplimiento de sus } \\
\text { obligaciones tributarias (gastos de impresión, gastos } \\
\text { computacionales, trámites en el banco, capacitaciones, } \\
\text { movilizaciones, etc.)? }\end{array}$ & 2,10 & 6,05 & 230 \\
\hline $\begin{array}{l}\text { P15. ¿Cuál es el costo anual del personal encargado de } \\
\text { efectuar las actividades de los enunciados antes } \\
\text { mencionados? }\end{array}$ & 1,23 & 5,81 & 230 \\
\hline
\end{tabular}

Nota: elaborado en base a la encuesta aplicada a las medianas empresas de Riobamba, año 2021

\section{Costos Externos}

Los costos de cumplimento también están conformados por los costos externos, que permiten analizar dichos costos incurridos por las medianas empresas de la ciudad de Riobamba, en el año 2019. Como se puede evidenciar en la tabla 5: 


\section{Tabla 5}

\section{Costos Externos}

\begin{tabular}{lccc}
\hline \multicolumn{1}{c}{ Pregunta } & Media & $\begin{array}{c}\text { Desviación } \\
\text { Estándar }\end{array}$ & $\begin{array}{c}\text { No. de } \\
\text { Análisis }\end{array}$ \\
\hline $\begin{array}{l}\text { P1. ¿Cuál es el medio utilizado para el registro de sus } \\
\text { operaciones tributarias y contables? }\end{array}$ & 2,00 & 0,000 & 230 \\
$\begin{array}{l}\text { P2. Para procesar los libros contables, ¿utiliza un } \\
\text { software contable y cuánto fue el costo? }\end{array}$ & 1,16 & 4,30 & 230 \\
$\begin{array}{l}\text { P3. ¿Durante los últimos meses tuvo que contratar a un } \\
\text { profesional o asesor contable externo para el }\end{array}$ & 1,14 & 3,47 & 230 \\
$\begin{array}{l}\text { cumplimiento de sus obligaciones tributarias? } \\
\text { P4. ¿En el caso de su respuesta ser afirmativa, cuál fue }\end{array}$ & 2,15 & 8,79 & 230 \\
el costo anual incurrido en la contratación de dicho & & & \\
profesional o asesor contable?
\end{tabular}

Nota: elaborado en base a la encuesta aplicada a las medianas empresas de Riobamba, año 2021

\section{Medición de costo de transacción tributaria}

\section{Datos de Costos de Cumplimiento}

Considerando que los Costos de cumplimiento son el resultado de la suma de los Costos Internos y los Costos Externos, los cuales permiten conocer los costos incurridos por las medianas empresas, tal como se presenta a continuación:

\section{a. Costos internos}

Los Costos Internos están conformados por una serie de actividades realizadas por las medianas empresas y el tiempo que las mismas conlleva realizarlas, dando un promedio de 2.408 horas al año, tal como se muestra en la tabla 6, de igual forma en la tabla 7 se evidencia el costo de dichas actividades, dando como resultado un costo promedio de $\$ 12.200,00$ y finalmente los gastos administrativos que se presentan en la tabla 8 , los cuales son un total de $\$ 3.000,00$.

\section{Tabla 6}

Tiempo de las actividades desarrolladas por los contribuyentes

\begin{tabular}{lll}
\hline No & ACTIVIDAD & TIEMPO \\
\hline 1 & Inscripción y obtención del Registro Único de Contribuyentes & 2 horas \\
\hline
\end{tabular}




\section{Tabla 6}

Tiempo de las actividades desarrolladas por los contribuyentes (continuación)

\begin{tabular}{lll}
\hline No & ACTIVIDAD & TIEMPO \\
\hline 2 & Registro de sus operaciones contables & 1.920 horas \\
3 & Cálculo y declaración de sus impuestos & 450 horas \\
4 & Pago de sus impuestos & 12 horas \\
5 & Acción de control por parte del SRI & 12 horas \\
6 & Realización de reclamo o devolución de impuestos & 12 horas \\
\hline TOTALES & 2.408 horas
\end{tabular}

Nota: elaborado en base a la encuesta aplicada a las medianas empresas de Riobamba, año 2021

Tabla 7

Costo del tiempo de las actividades desarrolladas por los contribuyentes

\begin{tabular}{lll}
\hline No & ACTIVIDAD & VALOR \\
\hline 1 & Gasto notarial al constituir su empresa & 600,00 \\
2 & $\begin{array}{l}\text { Impresión de C/P (facturas, notas de venta, guía de remisión, } \\
\text { notas de crédito y débito y liquidaciones) por millar }\end{array}$ & 800,00 \\
3 & $\begin{array}{l}\text { Costo anual del personal encargado de efectuar las actividades } \\
\text { de los enunciados antes mencionados }\end{array}$ & $10.800,00$ \\
\hline
\end{tabular}

TOTALES

$12.200,00$

Nota: elaborado en base a la encuesta aplicada a las medianas empresas de Riobamba, año 2021

Gastos Administrativos

Tabla 8

Costo de los gastos administrativos

\begin{tabular}{lll}
\hline No & \multicolumn{1}{c}{ ACTIVIDAD } & VALOR \\
\hline 1 & $\begin{array}{l}\text { Gastos administrativos anuales que se producen en su empresa para el } \\
\text { cumplimiento de sus obligaciones tributarias (gastos de impresión, }\end{array}$ & \\
& $\begin{array}{l}\text { gastos computacionales, trámites en el banco, capacitaciones, } \\
\text { movilizaciones, etc.) }\end{array}$ & \\
& & $3.000,00$ \\
\hline TOTALES
\end{tabular}

Nota: elaborado en base a la encuesta aplicada a las medianas empresas de Riobamba, año 2021 


\section{b. Costos externos}

En cuanto, a los costos externos son aquellas actividades que efectúan los contribuyentes de manera externa para el cumplimiento de sus obligaciones tributarias, tal y como se muestra en la tabla 9.

\section{Tabla 9}

Costos externos de los contribuyentes

\begin{tabular}{lll}
\hline No. & ACTIVIDAD & VALOR \\
\hline 1 & Adquisición de Software Contable & $8.000,00$ \\
2 & Contratación de Asesor contable externo & $6.500,00$ \\
\hline \multicolumn{2}{l}{ TOTALES } & \\
\hline
\end{tabular}

Nota: elaborado en base a la encuesta aplicada a las medianas empresas de Riobamba, año 2021

Datos de costos administrativos

Según la información otorgada por el Servicio de Rentas Internas (2021), los siguientes datos corresponden a funcionarios en el año 2019, en la ciudad de Riobamba.

\section{Tabla 10}

Funcionarios del Servicio de Rentas Riobamba, periodo 2019

\begin{tabular}{lc}
\hline Departamentos & Número de servidores año 2019 \\
\hline Dirección Provincial & 2 \\
Asistencia al Contribuyente & 17 \\
Auditoría Tributaria & 10 \\
Cobro & 8 \\
Gestión Tributaria & 23 \\
Jurídico & 2 \\
Planificación y Control de gestión & 1 \\
Reclamos & 3 \\
Soporte Operacional & 14 \\
\hline \multicolumn{2}{c}{ TOTAL } \\
\hline
\end{tabular}

Fuente: Servicio de Rentas Internas (2021) 
Aplicación de procedimiento de la distribución de los costos administrativos

Para el cálculo de los Costos Administrativos se considera aquellas cuentas principales del presupuesto de la Administración Tributaria, así como de las principales actividades ofrecidas al contribuyente, siguiendo todo el procedimiento de la estimación y distribución de estos costos, como se presenta a continuación:

\section{Presupuesto ejecutado}

\section{Tabla 11}

Presupuesto ejecutado del periodo 2019

\begin{tabular}{llr}
\hline \multicolumn{1}{c}{ PARTIDAS } & & AÑO 2019 \\
\hline Remuneraciones & $\$$ & $2.436 .768,00$ \\
Bienes y Servicios de Consumo e Inversión & $\$$ & $1.517 .064,00$ \\
Bienes de Larga Duración & $\$$ & $68.339,00$ \\
Transferencias y Donaciones Corrientes & $\$$ & $3.577,98$ \\
Otros Gastos & $\$$ & $32.917,00$ \\
Total & $\$$ & $4.058 .665,98$ \\
\hline \multicolumn{2}{c}{ Nota: Elaborado en base a información del SRI, (2021) }
\end{tabular}

Capacidad instalada por procesos principales

Tabla 12

Capacidad instalada por procesos principales

\begin{tabular}{llll}
\hline Centros de costos & Funcionarios & $\begin{array}{c}\text { Días anuales de } \\
\text { trabajo }\end{array}$ & $\begin{array}{c}\text { Porcentajes capacidad } \\
\text { instalada }\end{array}$ \\
\hline Asistencia al Contribuyente & 17 & 4.250 & 26,98 \\
Gestión Tributaria & 23 & 5.750 & 36,51 \\
Cobranza & 8 & 2.000 & 12,70 \\
Reclamos & 3 & 750 & 4,76 \\
Jurídico & 2 & 500 & 3,17 \\
Auditoría & 10 & 2.500 & 15,87 \\
\hline TOTAL & 63 & 15.750 & 100 \\
\hline Días laborables al año & 250 & & \\
\hline
\end{tabular}

Nota: Elaborado en base a información del SRI, (2021) 


\section{VPDigital}

Cálculo de los costos administrativos por procesos clave

\section{Tabla 13}

\section{Cálculo de los costos administrativos por procesos}

\begin{tabular}{|c|c|c|c|c|c|c|}
\hline \multirow[b]{2}{*}{ Centros de costos } & \multirow[b]{2}{*}{$\begin{array}{c}\text { Porcentajes } \\
\text { capacidad } \\
\text { instalada }\end{array}$} & \multirow[b]{2}{*}{$\begin{array}{c}\text { Total gastos } \\
\text { administrativos } \\
(\mathrm{CA}=\mathrm{b}+\mathrm{c})\end{array}$} & \multirow[b]{2}{*}{$\begin{array}{l}\text { Total gastos } \\
\text { directos } \\
\text { corrientes } \\
\text { (b) }\end{array}$} & \multirow[b]{2}{*}{$\begin{array}{l}\text { Total gastos } \\
\text { indirectos } \\
\text { (c) }\end{array}$} & \multicolumn{2}{|c|}{ Gastos indirectos } \\
\hline & & & & & $\begin{array}{l}\text { Gastos corrientes } \\
\text { en remuneración y } \\
\text { administración } \\
\text { general }\end{array}$ & $\begin{array}{l}\text { Inversión en } \\
\text { infraestructura }\end{array}$ \\
\hline Asistencia al Contribuyente & 26,98 & $\$ 1.066 .529,53$ & $\$ 507.960,00$ & $\$ 558.569,53$ & $\$ 557.604,04$ & $\$ 965,49$ \\
\hline Gestión Tributaria & 36,51 & $\$ 1.523 .819,72$ & $\$ 768.108,00$ & $\$ 755.711,72$ & $\$ 754.405,47$ & $\$ 1.306,25$ \\
\hline Cobranza & 12,70 & $\$ 530.984,25$ & $\$ 268.128,00$ & $\$ 262.856,25$ & $\$ 262.401,90$ & $\$ 454,35$ \\
\hline Reclamos & 4,76 & $\$ 157.143,09$ & $\$ 58.572,00$ & $\$ 98.571,09$ & $\$ 98.400,71$ & $\$ 170,38$ \\
\hline Jurídico & 3,17 & $\$ 118.538,06$ & $\$ 52.824,00$ & $\$ \quad 65.714,06$ & $\$ 65.600,48$ & $\$ 113,59$ \\
\hline Auditoría & 15,87 & $\$ 596.890,31$ & $\$ 268.320,00$ & $\$ 328.570,31$ & $\$ 328.002,38$ & $\$ 567,93$ \\
\hline TOTAL & 100 & $\$ 3.993 .904,96$ & $\$ 1.923 .912,00$ & $\$ 2.069 .992,96$ & $\$ 2.066 .414,98$ & $\$ 3.577,98$ \\
\hline
\end{tabular}

Nota: Elaborado en base a información del SRI, (2021)

Aplicación de las fórmulas

A continuación, se muestra los cálculos efectuados para la obtención de los costos de cumplimiento, lo cual corresponde a los costos de cumplimiento incurridos por las medianas empresas, obteniendo un total de 2.408 horas, el cual tiene un costo de $\$ 5.066445183$, los gastos administrativos corresponden a $\$ 3.000,00$; dando como resultado un total de $\$ 12.508 .56$ de costos internos.

El costo externo da como resultado un total de $\$ 14.500,000$. Es así, que aplicado dicha fórmula tenemos que las Medianas Empresas tienen un costo de cumplimiento de \$29.708.56 anuales, tal como se presenta a continuación:

$$
\begin{aligned}
& \text { Costos Internos }=(\text { Tiempo } * \text { Valor del tiempo })+\text { Gastos Administrativos } \\
& \text { DATOS: }
\end{aligned}
$$

Tiempo $=2.408$ horas

Valor del Tiempo $=12.200 / 2.408=5.066445183$

Gastos Administrativos $=3.000$ 


$$
\begin{gathered}
C I=(2.408 * 5,07)+3.000 \\
C I=12.208,56+3.000 \\
C I=15.208,56 \\
\text { Costos de Cumplimiento }=\text { Costos Internos }+ \text { Costos Externos } \\
C C=15.208,56+14.500 \\
C C=29.708,56
\end{gathered}
$$

\section{Costos de transacción tributaria}

Con la determinación de los Costos de Cumplimiento que corresponde a $\$ 29.708,56$ y los Costos Administrativos de \$3`993.904,96 como se puede evidenciar en la tabla 12, por lo tanto, se procede a la determinación del Costo de Transacción Tributaria de las medianas empresas de la ciudad de Riobamba en el año 2019, lo cual nos da un total de $\$ 4.023 .613,52$ anuales.

$$
\begin{gathered}
\boldsymbol{C T T}=\boldsymbol{C A}+\boldsymbol{C C} \\
C T T=3^{\prime} 993.904,96+29.708,56 \\
C T T=4.023 .613,52
\end{gathered}
$$

\section{Comprobación de la hipótesis}

Para la demostración de la Hipótesis General de la investigación, se aplicó el modelo de regresión lineal considerando los dos componentes principales de la Transacción Tributaria como son los Costos Administrativos y Costos de Cumplimiento, siendo el Modelo 1, de igual manera con el fin de verificar como contribuyen los elementos de los Costos Administrativos los cuales comprende como variables a los Gastos Directos y a los Gastos Indirectos, se utilizó el Modelo 2, y finalmente se aplicó el Modelo 3, que comprende a los componentes de los Costos de Cumplimiento los cuales comprende como variables a los Costos Internos y Costos Externos.

Los resultados obtenidos de la investigación demuestran que la Medición de la Transacción Tributaria incide favorablemente en las Medianas empresas de la ciudad de Riobamba en un 42,9\% $\left(\mathrm{R}^{2}=0.429\right)$, con una correlación de $65,5 \%(\mathrm{R}=0.655)$, asimismo se demuestra que los Costos Administrativos realizados por la Administración Tributaria y los Costos de Cumplimiento efectuados por los Contribuyentes inciden en el cumplimiento de las obligaciones tributarias y contables de las medianas empresas, en un $100 \%\left(\mathrm{R}^{2}=1,00\right)$ y una correlación de $100 \%(\mathrm{R}=1,00)$, lo cual genera que la recaudación de impuestos por parte del Servicio de Rentas Internas sea eficiente, de igual forma que los contribuyentes cumplen correctamente sus obligaciones tributarias. 


\section{VPDigital}

\section{Tabla 14}

Aplicación de Modelo de regresión lineal

\begin{tabular}{|c|c|c|c|c|}
\hline \multicolumn{5}{|l|}{ RESUMEN DEL MODELO } \\
\hline Modelo & $\mathrm{R}$ & $\mathrm{R}$ cuadrado & $\begin{array}{l}\mathrm{R} \quad \text { cuadrado } \\
\text { corregida }\end{array}$ & $\begin{array}{l}\text { Error tipo. de la } \\
\text { estimación }\end{array}$ \\
\hline Modelo 1 & & & & \\
\hline $\begin{array}{l}\text { Variable dependiente: COSTOS } \\
\text { ADMINISTRATIVOS (Gastos } \\
\text { Directos + Gastos Indirectos) }\end{array}$ &, $655^{\mathrm{a}}$ & 429 & ,286 & 1,58114 \\
\hline
\end{tabular}

\section{Tabla 15}

Aplicación de Modelo de regresión lineal

\section{RESUMEN DEL MODELO}

\begin{tabular}{l|l|l|l|l}
\hline Modelo & $\mathrm{R}$ & $\mathrm{R}$ cuadrado & $\begin{array}{l}\mathrm{R} \text { cuadrado } \\
\text { corregida }\end{array}$ & $\begin{array}{l}\text { Error tipo. de la } \\
\text { estimación }\end{array}$ \\
\hline Modelo 2 & $1,000^{\mathrm{a}}$ & 1,000 & 1,000 & 0,00000 \\
$\begin{array}{l}\text { Variable dependiente: GASTOS } \\
\text { DIRECTOS }\end{array}$ & & & & \\
\hline $\begin{array}{l}\text { Variables predictoras: (Constante), } \\
\text { GASTOS INDIRECTOS }\end{array}$ & & & 1,000 & 0,00000 \\
\hline $\begin{array}{l}\text { Modelo 3 COSTOS } \\
\text { Variable dependiente: C,000 }\end{array}$ & 1,000 & & \\
INTERNOS & & & & \\
\hline $\begin{array}{l}\text { Variables predictoras: (Constante), } \\
\text { COSTOS EXTERNOS }\end{array}$ & & & & \\
\hline
\end{tabular}

Nota: elaborado en base a la encuesta aplicada a las medianas empresas de Riobamba, año 2021

\section{Conclusiones}

- Se llega a la conclusión que la utilización de la metodología de medición de costo de transacción tributaria es una buena herramienta para que la Administración Tributaria Central pueda evaluar los costos que incurren los contribuyentes y el Servicio de Rentas Internas.

- La contratación de los asesores contables y tributarios externos en las empresas se ha convertido en una práctica muy común por parte de las entidades, por lo 
tanto, la Administración Tributaria debería poner más atención a esta situación, no con la finalidad de limitar dicha práctica sino más bien que estos agentes sean el vínculo con el contribuyente y lograr una relación más efectiva.

- A través de la aplicación de la metodología de la medición del costo de transacción tributaria en las medianas empresas, se ha logrado determinar que efectivamente dicha medición incide favorablemente en dichas empresas, puesto que la Administración Tributaria conoce los procesos que están siendo de mayor gasto para los contribuyentes y tomar las correspondientes decisiones para reducir dichos procesos, por lo tanto, se generaría un ahorro para los contribuyentes al momento de realizar todo el proceso de cumplimiento de sus obligaciones tributarias.

\section{Referencias Bibliográficas}

Arredondo, M. (2015). Contabilidad y análisis de costos. México: Grupo Editorial Patria S.A. .

Castillo , D., \& Vásquez, F. (2015). Influencia del costo de transacción tributario en la informalidad de las Mypes comerciantes minoristas. Revista científica In Crescendo Ciencias Contables \& Administrativas.

Centro Inter-americano de Naciones Unidas. (2015). Medición de los Costos de Transacción Tributarios en Pequeñas y Medianas Empresas. New York: Naciones Unidas.

Código Orgánico de la Producción, C. e. (20 de mayo de 2020). Dirección Nacional Jurídica. Obtenido de Del Fomento y Desarrollo de las MIPYMES: https://www.sri.gob.ec/BibliotecaPortlet/descargar/4cdfe82a-6702-4147-917e344eb79d8eae/CO

Código Tributario. (2019). Deberes Formales del Contribuyente o responsable. Quito: Dirección Nacional Jurídica. Departamento de Normativa Tributaria.

Gutiérrez, R. (2016). Introducción al Método Científico. México: Esfinge.

Laporta, R. (2016). Costos y gestión empresarial. Colombia: Ecoe Ediciones.

López, P. (2015). Población muestra y muestreo. Perú: Punto cero.

Mera, E., Vargas, G., \& Flores, S. (2017). El costo de la transacción tributaria en las empresas comerciales de la Provincia de Tungurahua. Revista Científica Hermes, 21-22. 
Montás, G. (junio de 15 de 2018). Centro Regional de Estrategías Económicas Sostenibles. Obtenido de Eficiencia y costos de cumplimiento tributario: http://www.crees.org.do/es/noticia/eficiencia-y-costos-de-cumplimientotributario

Servicio de Rentas Internas. (20 de Septiembre de 2021). Obtenido de https://www.sri.gob.ec/bases-legales

Rodríguez, A., \& Pérez, A. (2017). Métodos científicos de indagación y de construcción del conocimiento. Cuba: Revista Escuela de Administración de Negocios.

Vargas, G. (2017). El costo de la transacción tributaria y el cumplimiento de las obligaciones fiscales en las pequeñas y medianas empresas, sector comercio de la provincia de Tungurahua. Ambato: Universidad Técnica de Ambato.

\section{Ciencia LDigital}


El artículo que se publica es de exclusiva responsabilidad de los autores y no necesariamente reflejan el pensamiento de la Revista Visionario Digital.

\section{¿Ciencia}

El artículo queda en propiedad de la revista y, por tanto, su publicación parcial y/o total en otro medio tiene que ser autorizado por el director de la Revista Visionario Digital.
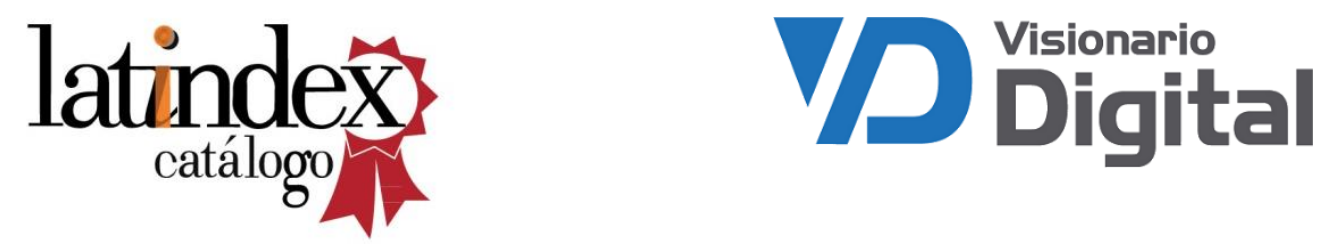

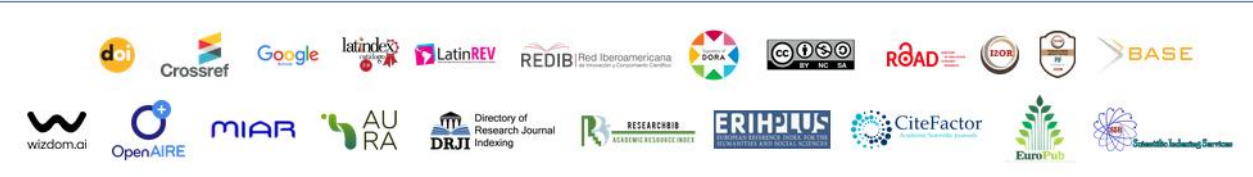

